\title{
Analytic Formulation of the Kinestatics of Robot Manipulators with Arbitrary Topology*
}

\author{
E. Staffetti \\ Katholieke Universiteit Leuven \\ Department of Mechanical Engineering \\ Celestijnenlaan 300B, 3001 Heverlee, Belgium \\ Ernesto.Staffetti@mech.kuleuven.ac.be
}

\author{
F. Thomas \\ Institut de Robòtica i Informàtica Industrial \\ CSIC-UPC \\ Llorens i Artigas 4-6, 08028 Barcelona, Spain \\ fthomas@iri.upc.es
}

\begin{abstract}
In this paper an analytic formulation of the statics and the instantaneous kinematics of robot manipulators based on the Grassmann-Cayley algebra is presented. The notions of twist, wrench, twist space and wrench space are mathematically represented by the concept of extensors of this algebra and the reciprocity relation between twist and wrench spaces of partially constrained rigid bodies is reflected by its inherent duality. Kinestatic analysis of manipulators implies the computation of sums and intersections of the twist and wrench spaces of the composing chains which are carried out by means of the join and meet operators of this algebra when the linear subspaces involved in the kinestatic analysis of manipulators are represented by extensors. The importance of the Grassmann-Cayley algebra in kinestatics is that it has an explicit formula for the meet operator that gives analytical expressions of the twist and wrench space of robot manipulators with arbitrary topology.
\end{abstract}

\section{Introduction}

The theorems of Chasles and Poinsot state, respectively, that any instantaneous velocity of a rigid body can be described as a twist on a screw and that any set of forces and moments that act on a rigid body can be described by means of a wrench on a screw. Relevant references about screw theory are [1], in which a comprehensive work on this subject was presented for the first time, and [?], where a modern

*This work has been partially supported by the K. U. Leuven's Concerted Research Action GOA 99/04 and by the Spanish CICYT under contract TIC2000-0696 presentation of this theory is given and applications to mechanisms and robot manipulators are reported.

Any possible twist of a rigid body partially constrained by a wrench is characterized by the reciprocity condition, that is, the work generated by any twist against any wrench must be zero and the term kinestatics refers to this dualistic relation between the statics and the instantaneous kinematics of rigid bodies. The concept of reciprocity was established for the fist time in [1] and mathematical frameworks of differential and projective geometry have been both used to describe this condition, also called duality. Paradoxically, as clearly pointed out in [3], the well established concept of reciprocity between twist and wrench spaces of partially constrained rigid bodies has been ignored in many works and confused with the concept of orthogonal complement.

In this paper we gain a deeper understanding of the projective approach to the kinestatics of robot manipulators with arbitrary topology using the formalism of the Grassmann-Cayley algebra, extending the method presented in [4] for the kinestatic analysis of serial and parallel manipulators by introducing over the graph of kinematic constraints of the manipulator the delta-wye transformation which permits the analysis of those configuration that cannot be carried out with only series and parallel reductions.

It will be shown that the Grassmann-Cayley algebra permits the mathematical representation of all the basic concepts of screw theory. In particular, twist and wrench spaces of partially constrained rigid bodies can be described using the notion of extensor of this algebra and the reciprocity relation between them is reflected by its inherent, duality.

Kinestatic analysis of manipulators requires the 
computation of sums and intersection of the twist and wrench spaces of the composing kinematic chains, operations that can be implemented by means of the join and meet operators of the GrassmannCayley algebra when these spaces are represented by means of extensors. In particular, the problem of the intersection of linear subspace arises in the statics of serial manipulators and in the instantaneous kinematics of parallel manipulators. While analytic formulations of the instantaneous kinematics of parallel manipulators have been derived $[5,6]$ an analytic formulation of both statics and instantaneous kinematics of manipulators with arbitrary topology is not present in the literature.

The importance of the Grassmann-Cayley is that it has an explicit formula for the meet which gives analytical, coordinate-free expressions for the intersection of linear subspaces involved in the kinestatic analysis of robot manipulators. This is a clear advantage with respect to common linear algebra-based techniques that give only algorithmic relations between the basis vectors of two linear subspaces and the basis vectors of their intersection which, therefore, are coordinate-dependent.

The results of the kinestatic analysis of general kinematic chains can be directly applied to the analysis of rigid body interactions once the kinematic chain instantaneous equivalent to the contacts between a moving object and a static environment has been determined. In [7] the Grassmann-Cayley algebra has been applied to the analysis of rigid body interactions for compliant motion tasks. A comprehensive study of the possible applications of the Grassmann-Cayley algebra and other methods derived from invariant theory to robotics is [ 8$]$.

The next section reviews the most relevant properties of a concrete version of the GrassmannCayley algebra based on the Plücker coordinates of linear subspaces of a given vector space and those of their duals, also called dual Plücker coordinates. In Section 3 the representation of generalized forces and velocities in the Grassmann-Cayley algebra setting is explained. In Section 4 a method for the kinestatic analysis of kinematic chains with arbitrary topology is presented. It is based on series and parallel reductions and delta-wye transformations of the graph that represents the kinematic constraints of the robot manipulator. Another operation, the deltawye transformations, is introduced for the study of those cases that cannot be solved using only serial and parallel reductions. Finally, Section 5 contains the conclusions.

\section{The Grassmann-Cayley Algebra}

In this section we report the most relevant properties of a version of the Grassmann-Cayley algebra that involves Plücker coordinates. Further details can be found in [9].

The Grassmann-Cayley algebra is essentially the Grassmann algebra without scalar product and can be also viewed as the exterior algebra with duality. In [10] the representation of generalized forces and velocities using the exterior algebra is introduced and this formalism is applied to the study of the statics of bar-and-joint frameworks and the instantaneous kinematics of articulated panel structures. The work [11] is a comprehensive survey of the abstract properties of the Grassmann-Cayley algebra while [12] emphasizes the practical approach to it. In [9] some possible applications of this algebra to robotics are outlined, including the analysis of singular configurations and the instantaneous kinematics of manipulators.

If $\mathbf{x}=\left(x_{1}, x_{2}, \ldots, x_{m}\right)^{T}$ is a point in $\mathbb{R}^{m}$ given in terms of Cartesian coordinates, the vector $x=$ $\left(x_{1}, x_{2}, \ldots, x_{m}, 1\right)^{T}$ is defined to be its homogeneous coordinate vector. If we allow points with the last coordinate 0 for representing projective points at infinity, the standard $m$-dimensional projective space that includes $\mathbb{R}^{m}$ is obtained. So, we represent $\mathbb{R}^{m}$ with points at infinity by a $(m+1)$-dimensional vector space $V$.

\subsection{Plücker Coordinates and Dual Plücker Coordinates}

Let $U$ be a $k$-dimensional subspace of the $(m+1)$ dimensional vector space $V$, and $\left\{u_{1}, u_{2}, \ldots, u_{k}\right\}$ a basis of it. When these vectors are arranged as rows of a matrix, we obtain

$$
\left(\begin{array}{ccccc}
u_{1,1} & u_{1,2} & \cdots & u_{1, m} & 1 \\
u_{2,1} & u_{2,2} & \cdots & u_{2, m} & 1 \\
\cdots & \cdots & \cdots & \cdots & \cdots \\
u_{k, 1} & u_{k, 2} & \cdots & u_{k, m} & 1
\end{array}\right) .
$$

The $\left(j_{1}, j_{2}, \cdots, j_{k}\right)$-th Plücker coordinate of the subspace $U$, denoted by $P_{j_{1}, j_{2}, \cdots, j_{k}}$, is the $k \times k$ determinant formed by the $k$ columns of the above matrix with indices $j_{1}, j_{2}, \cdots, j_{k}$. Since we have a Plücker coordinate for each combination of the $k$ columns, the total number of Plücker coordinates is $\left(\begin{array}{c}m+1 \\ k\end{array}\right)$. The Plücker coordinate vector of the subspace $U$ is the vector $P_{U}$ that contains in some predetermined order its Plücker coordinates. $U$ uniquely determines $P_{U}$ up to a scalar multiple. 
The Plücker coordinate vector of the line $\Lambda$ passing through points $a$ and $b$, represented in homogeneous coordinates by the rows of

$$
\left(\begin{array}{llll}
a_{1} & a_{2} & a_{3} & 1 \\
b_{1} & b_{2} & b_{3} & 1
\end{array}\right)
$$

is defined as

$$
\begin{gathered}
P_{\Lambda}=\left(-P_{1,4},-P_{2,4},-P_{3,4}, P_{2,3},-P_{1,3}, P_{1,2}\right)^{T}= \\
\left(b_{1}-a_{1}, b_{2}-a_{2}, b_{3}-a_{3}, a_{2} b_{3}-a_{3} b_{2}, a_{3} b_{1}-a_{1} b_{3}, a_{1} b_{2}-a_{2} b_{1}\right)^{T}=
\end{gathered}
$$

$$
(\mathbf{S}, \mathbf{r} \times \mathbf{S})^{T}
$$

where $\mathbf{S}=\mathbf{b}-\mathbf{a},(\mathbf{r} \times \mathbf{S})$ represents the moment of $\Lambda$ with respect to the origin, and $\mathbf{r}$ is any point on the line. The vector $P_{\Lambda}$ is determined by $\Lambda$ up to a scalar multiple and, since it will be used to represent rotations and forces, it is convenient to normalize it such that $\mathbf{S} \cdot \mathbf{S}=1$.

The point at infinity on $\Lambda$ has homogeneous coordinates $\left(b_{1}-a_{1}, b_{2}-a_{2}, b_{3}-a_{3}, 0\right)^{T}$ and can be thought of as infinitely far away in the direction given by $\mathbf{S}$.

A line at infinity is determined by two distinct points at infinity, $c$ and $d$, represented by the rows of

$$
\left(\begin{array}{llll}
c_{1} & c_{2} & c_{3} & 0 \\
d_{1} & d_{2} & d_{3} & 0
\end{array}\right)
$$

and its Plücker coordinate vector is

$$
\begin{array}{r}
P_{\Lambda}=\left(0,0,0, c_{2} d_{3}-c_{3} d_{2}, c_{3} d_{1}-c_{1} d_{3}, c_{1} d_{2}-c_{2} d_{1}\right)^{T}= \\
(0,0,0, \mathbf{r} \times \mathbf{S})^{T}
\end{array}
$$

Since lines at infinity will be used to represent translations and moments, it is convenient to normalize this vector in such a way that $(\mathbf{r} \times \mathbf{S}) \cdot(\mathbf{r} \times$ S) $=1$.

The Plücker coordinate vector of the plane $\Pi$, determined by three finite points whose homogeneous coordinates are the rows of

$$
\left(\begin{array}{llll}
a_{1} & a_{2} & a_{3} & 1 \\
b_{1} & b_{2} & b_{3} & 1 \\
p_{1} & p_{2} & p_{3} & 1
\end{array}\right)
$$

is defined as

$$
P_{\Pi}=\left(P_{2,3,4},-P_{1,3,4}, P_{1,2,4}, P_{1,2,3}\right)^{T}=(\mathbf{N}, \mathbf{r} \cdot \mathbf{N})^{T},
$$

where $\mathbf{N}$ is a normal vector to $\Pi$ and $\mathbf{r}$ is the position vector of any point on $\Pi$.

The subspace $U$ can be represented as the subspace spanned by the $k$ basis vectors or as the intersection of $(n-k)$ hyperplanes. This second representation permits to define for each subspace $U$ a dual subspace $U^{*}$ as explained in [13, Vol. I, Book II, Chap. V, Part 5] A line in $\mathbb{R}^{3}$, for example, can be constructed in two ways as the join of two points or as the meet of two planes. Traditionally, the Plücker coordinates of lines are called ray coordinates while the dual Plücker coordinates are called axis coordinates of the line.

Thus, the dual Plücker coordinate vector of $U$ is defined to be the vector that contains the Plücker coordinates of $U^{*}$ called dual Plücker coordinates of $U$. It can be proved that the number of dual Plücker coordinates equals the number of Plücker coordinates, the only difference between them being their ordering and some sign changes. For example, the Plücker and dual Plücker coordinate vectors of lines in $\mathbb{R}^{3}$ have the first three and the last three elements interchanged.

\subsection{The Join and Meet Operators}

Let $V$ be an $n$-dimensional vector space over the field $\mathbb{R}, U$ a $k$-dimensional subspace of $V$, and $\left\{u_{1}, u_{2}, \ldots, u_{k}\right\}$ a basis of it. Let us consider the Plücker coordinate vector of $U$ denoted by

$$
P=u_{1} \vee u_{2} \vee \ldots \vee u_{k} .
$$

This vector, that can be regarded as a vector in the $\left(\begin{array}{l}n \\ k\end{array}\right)$-dimensional vector space $V^{(k)}$, is called $k$-extensor or decomposable $k$-tensor.

The subspace $U$, also denoted as $\bar{P}$, is defined as the support of the extensor $P$ and the scalar $k$ is defined to be the step of the extensor. Elements of $V^{(k)}$ that are not $k$-extensors are called indecomposable $k$-tensors and can always be expressed as linear combination of $k$-extensors. The term antisymmetric $k$-tensor refers to both decomposable and indecomposable $k$-tensors while it is called tensor a linear combination of $k$ tensors having different steps.

Let $a_{1} \vee a_{2} \vee \ldots \vee a_{k}$ and $B=b_{1} \vee b_{2} \vee \ldots \vee b_{j}$ be two extensors of steps $k$ and $j$, respectively. The join of $A$ and $B$ is defined as the $(j+k)$-extensor

$$
A \vee B=a_{1} \vee a_{2} \vee \ldots \vee a_{k} \vee b_{1} \vee b_{2} \vee \ldots \vee b_{j} .
$$

If the vectors $\left\{a_{1}, a_{2}, \ldots, a_{k}, b_{1}, b_{2}, \ldots, b_{j}\right\}$ are linearly dependent, then $A \vee B=0$. If they are independent,

$$
\overline{A \vee B}=\bar{A}+\bar{B}=\operatorname{span}(\bar{A} \cup \bar{B}) .
$$

This means that the join of two extensors represent the operation of joining the associated vector subspaces. The joint operator is anticommutative. If $A$ and $B$ are extensors of step $k$ and $j$ respectively, then $A \vee B=(-1)^{k j} B \vee A$. 
We have defined the join of extensor but, since each tensor can be expressed as linear combination of extensors, the join of tensors can be reduced to the join of extensors by assuming distributivity of the join over the sum.

The vector space $V^{(k)}$ is closed under the addition but it is not closed under the join operation. We combine the vector spaces $V^{(k)}$ into other vector space over $\mathbb{R}$ obtained as the direct sum of the $V^{(k)}$ for $k=1,2, \ldots, n$, which is closed under both operations, that is,

$$
\Lambda(V)=V^{(0)} \oplus V^{(1)} \oplus \cdots \oplus V^{(n)} .
$$

The vector space $\Lambda(V)$ with the join operation is known as the exterior algebra over $V$.

Let $A$ and $B$ be the above two extensors with $(j+k) \geq n$. The meet of $A$ and $B$ is the $(k+j-d)$ extensor defined by

$$
\begin{gathered}
A \wedge B=\sum_{\sigma} \operatorname{sgn}(\sigma)\left[a_{\sigma(1)}, \ldots, a_{\sigma(n-j)}, b_{1}, \ldots, b_{j}\right] \\
a_{\sigma(n-j+1)} \vee a_{\sigma(n-j+2)} \vee \cdots \vee a_{\sigma(k)}
\end{gathered}
$$

where the brackets stand for determinants and the sum is taken over all the permutations $\sigma$ of $\{1,2, \ldots, k\}$ such that $\sigma(1)<\sigma(2)<\cdots<\sigma(n-j)$ and $\sigma(n-j+1)<\sigma(n-j+2)<\cdots<\sigma(k)$. This formula, called the shuffle formula. It can be proved that if $A \neq 0$ and $B \neq 0$ and $\bar{A} \cup \bar{B}$ spans $V$, then

$$
\overline{A \wedge B}=\bar{A} \cap \bar{B} \text {. }
$$

Also the meet operator is anticommutative. If $A$ and $B$ are extensors of step $k$ and $j$ respectively, then $A \wedge B=(-1)^{(n-k)(n-j)} B \wedge A$.

Another important property of the $V$ and $\wedge$ operators is that they are dual operators. If we interchange $\vee$ and $\wedge$ we must interchange $V^{(k)}$ with $V^{*(n-k)}$. This gives the Grassmann-Cayley algebra properties analogous to those of a Boolean algebra. For example, we have that

$$
\begin{aligned}
& (A \vee B)^{*}=A^{*} \wedge B^{*}, \\
& (A \wedge B)^{*}=A^{*} \vee B^{*},
\end{aligned}
$$

and, if $\mathrm{A}$ is a $k$-extensor,

$$
\left(A^{*}\right)^{*}=(-1)^{k(n-k)} A .
$$

The Grassmann-Cayley algebra on $V$ is defined as the vector space $\Lambda(V)$ with the operations $V$ and $\wedge$. These operations are both associative, distributive over addition, and anticommutative.
The usefulness of this framework for the kinestatic analysis of manipulators comes from the fact that using the concept of extensor and the operators Grassmann-Cayley algebra we obtain purely symbolic expressions for the twist and wrench spaces which are relative invariants. This means that, if a non-singular change of basis of determinant $\rho$ is applied, the scalar coefficients of the terms of the expressions change by a predictable power of $\rho$. Since twist and wrench space are defined up to a scalar multiple we can affirm that these expressions are coordinate-free.

Of special relevance is the existence of an explicit formula for the meet operator which can be used to intersect the linear spaces involved in the kinestatic analysis of robot manipulators. This represents a clear advantage with respect to the classical methods for intersecting linear subspaces [14, Chap. 12] which are algorithmic, and therefore, coordinate-dependent.

\section{Screw Theory and Grassmann- Cayley Algebra}

If $a$ and $b$ are finite projective points, we can express a rotation in projective terms by the extensor $R=a \vee b$. Let us consider, for each point $p$ in space, the 3-extensor

$$
R \vee p=a \vee b \vee p=(\mathbf{v}, \mathbf{p} \cdot \mathbf{v})^{T},
$$

whose support is the plane defined by $a, b$, and $p$, and whose Plücker coordinate vector is a 4-tuple expressed by (2). The 2-extensor $R$, that represents the line passing through $a$ and $b$ expressed in Plücker coordinated by (1), is called center or axis of rotation whereas the free vector $\mathbf{S}=\mathbf{b}-\mathbf{a}$ can be interpreted as the angular velocity of rotation. We said in Section 2.1 that it is convenient to normalize the Plücker coordinate vectors by setting $\mathbf{S} \cdot \mathbf{S}=1$. In this way a multiplicative coefficient of this vector directly represents the angular velocity of rotation.

A translation can be described as a rotation about an axis at infinity with the corresponding Plücker coordinate vector normalized as explained in Section 2.1 .

With a serial composition of a translation and a rotations a more general screw motion in space can be obtained. This motion can be represented by a twist

$$
t=(\omega, \mathbf{v})^{T}
$$

A vector $\mathbf{f}=\left(f_{1}, f_{2}, f_{3}\right)^{T}$ that represents an Euclidean force applied at the Euclidean point $\mathbf{p}=$ 
$\left(p_{1}, p_{2}, p_{3}\right)^{T}$ can be represented in the projective space by the join of the two projective points $p=$ $\left(p_{1}, p_{2}, p_{3}, 1\right)^{T}$ and $f=\left(f_{1}, f_{2}, f_{3}, 0\right)^{T}$, that is, by the extensor

$$
F=p \vee f=(\mathbf{f}, \mathbf{p} \times \mathbf{f})^{T} .
$$

If we normalize this vector choosing $\mathbf{f} \cdot \mathbf{f}=1, \mathbf{a}$ multiplicative coefficient of this vector represents the intensity of the force.

If two forces $F_{1}=p \vee f$ and $F_{2}=q \vee g$ with $\mathbf{f}=-\mathbf{g}$ are applied to two distinct points $p$ and $q$, the resultant $G=F_{1}+F_{2}=p \vee f+q \vee g=$ $p \vee f+q \vee(-f)=(p-q) \vee f$ is called a couple. Since $p-q=\left(p_{1}-q_{1}, p_{2}-q_{2}, p_{3}-q_{3}, 0\right)^{T}$ is a point at infinity, a couple can be thought of as a force at infinity. We normalize the corresponding Plücker coordinate vector by setting $(\mathbf{p} \times \mathbf{f}) \cdot(\mathbf{p} \times \mathbf{f})=1$. The resultant of the composition of forces in space is a wrench

$$
w=(\mathbf{f}, \mathbf{m})^{T},
$$

where $f$ and $m$ are a force vector and a moment vector, respectively.

Twist and wrenches are in general indecomposable 2-tensors. We will represent twists using 2-tensors of $V^{(2)}$ while wrenches will be represented by means of the 2-tensors of its dual space $V^{*(2)}$.

\subsection{Reciprocity}

Let us consider a rigid body, say $M$, partially constrained by another, say $S$. We define the twist space $\mathbf{T}$ of $M$ as the vector space of all possible instantaneous twists that it can have with reference to $S$, and we define the wrench space $\mathbf{W}$ as the vector space of all reaction forces that can be generated in the interaction between $M$ and $S$.

In this situation any twist $t \in \mathbf{T}$ of $M$ must be reciprocal to any wrench $w \in \mathbf{W}$ between $M$ and $S$ because the power generated by $t$ and $w$ must be zero [3], that is,

$$
\boldsymbol{\omega} \cdot \mathbf{m}+\mathbf{v} \cdot \mathbf{f}=\mathbf{0}
$$

It is easy to see that this relation is nothing more than the expression of the pairing of the vector spaces $V^{(2)}$ and its dual $V^{*(2)}$. Thus, we can say that the twist and wrench spaces of a partially constrained rigid body are dual spaces in the sense of Section 2.1.

Now, it should be clear that the kinestatic analysis of robot manipulators can be done in terms of 2tensors, that is, in terms of the 6-dimensional vector spaces $V^{(2)}$ and $V^{*(2)}$.

If the twist space of a robot is the entire $V^{(2)}$, its end effector has full mobility while, if its wrench space is the whole $V^{*(2)}$, it can resist any wrench applied by the environment without exerting any force or torque at its joints. In general, since the twist space is a subspace of $V^{(2)}$ and the wrench space is a subspace of $V^{*(2)}$, it is convenient to set $V^{(2)}=H$ and work in the Grassmann-Cayley algebra $\Lambda(H)$ over this auxiliary vector space in which 2-extensors of $V^{(2)}$ become 6dimensional vectors.

\subsection{Composition of Kinematic Constraints}

It is well known that the twist (wrench) space of the serial (parallel) combination of motion constraints is the sum of the twist (wrench) spaces of the composing constraints. Analogously, the twist (wrench) space of the parallel (serial) combination of motion constraints is the intersection of the twist (wrench) spaces of the composing constraints [1.5]. It is important to point out that the reciprocity relation remains valid under serial and parallel combination of motion constraints.

The key point of our approach to the kinestatics is that once the twist and wrench spaces of the chains that compose a manipulator are represented by means of extensors their sum or intersection can be expressed and computed using the join and meet operators.

These considerations can be re-formulated in the language of the Grassmann-Cayley algebra for kinematic chains, for which the centers of motion of their links are called joint extensors. Indeed, from the properties (3) and (5) of the join and meet operators it follows that

- The twist (wrench) space of the serial (parallel) connection of kinematic chains is the support of the join of the extensors that represent the twist (wrench) spaces of the chains, provided that their twist (wrench) extensors are linearly independent.

- The twist (wrench) space of the parallel (serial) connection of kinematic chains is the support of the meet of the extensors that represent the twist (wrench) spaces of the chains, provided that the sum of the composing twist (wrench) spaces spans $H\left(H^{*}\right)$.

In the next section these considerations will be applied to the kinestatic analysis of robot manipulators with arbitrary topology.

\section{Kinestatic Analysis}

For the kinestatic analysis it is convenient to represent the topology of a robot manipulator using a 
directed graph, called graph of kinematic constraints, whose nodes stand for bodies and, if body $B_{p}$ is restricted in its motion with reference to body $B_{q}$, there will be a directed arc from $B_{p}$ to $B_{q}$ labelled . with the extensor $L_{p, q}=-L_{q, p}$.

Given two bodies $B_{s}$ and $B_{t}$ of a graph whose node are called terminals, which in general represent the basement and the end-effector of the robot, respectively, our problem is to find the equivalent constraint between them. This corresponds to reduce our graph to a single arc graph between the terminal nodes performing series and parallel reductions, and delta-wye transformations.

\subsection{Series and Parallel Reductions}

If the node that corresponds to the body $B_{i}$ is a nonterminal node of degree 2 between two arcs that are labelled with $L_{p, i}$ and $L_{i, q}$, we can remove this node and substitute the two arcs with another arc whose associated extensor is their join, that is, $L_{p, q}=L_{p, i} \vee L_{i, q}$.

Let $R_{\text {ser }}$ be a serial manipulator with $h \leq 6$ joints having linearly independent joint extensors $L_{0,1}, L_{1,2}, \ldots, L_{h-1, h}$, whose basement is represented by the body $B_{s}=B_{0}$ and the end effector by the body $B_{t}=B_{h}$. By applying the series reduction rule we have that the twist space of $R_{\text {ser }}$ is the support of the $h$-extensor $L_{R_{\text {ser }}}=L_{0,1} \vee L_{1,2} \vee \cdots \vee L_{h-1, h}$ of $H^{(h)}$, that is,

$$
\mathbf{T}_{R_{\text {ser }}}=\overline{L_{R_{\text {ser }}}}=\overline{L_{0,1} \vee L_{1,2} \vee \cdots \vee L_{h-1, h}},
$$

where $L_{i, i+1}$ are vectors of $H$.

The wrench space is its dual, that is, the support of the $(6-h)$-extensor $L_{R_{\text {ser }}}^{*}=\left(L_{0,1} \vee L_{1,2} \vee \cdots \vee\right.$ $\left.L_{h-1, h}\right)^{*}=L_{0,1}^{*} \wedge L_{1,2}^{*} \wedge \cdots \wedge L_{h-1, h}^{*}$ of $H^{*(6-h)}$, that is,

$$
\mathbf{W}_{R_{\text {ser }}}=\overline{L_{R_{s e r}}^{*}}=\overline{L_{0,1}^{*} \wedge L_{1,2}^{*} \wedge \cdots \wedge L_{h-1, h}^{*}} .
$$

In this expression, obtained by applying the property (6), the $L_{i, i+1}^{*}$ are 5-extensors of $H^{*(5)}$.

If $L_{p, q}^{1}$ and $L_{p, q}^{2}$ are two extensors that correspond to parallel arcs between the nodes $B_{p}$ and $B_{q}$ they can be replaced by a single arc whose extensor is their meet, that is $L_{p, q}=L_{p, q}^{1} \wedge L_{p, q}^{2}$.

Let $L_{0,1}^{1}, L_{0,1}^{2}, \ldots, L_{0,1}^{k}$ be the extensors that describe the twist spaces of the kinematic chains that compose a parallel manipulator $R_{p a r}$, in which $B_{s}=$ $B_{0}$ and $B_{t}=B_{1}$ represent the lower and the upper platforms, respectively. The wrench space of $R_{\text {par }}$ is

$$
\mathbf{W}_{R_{p a r}}=\overline{L_{R_{p a r}}^{*}}=\overline{\left(L_{0,1}^{1}\right)^{*} \vee\left(L_{0,1}^{2}\right)^{*} \vee \cdots \vee\left(L_{0,1}^{k}\right)^{*}}
$$

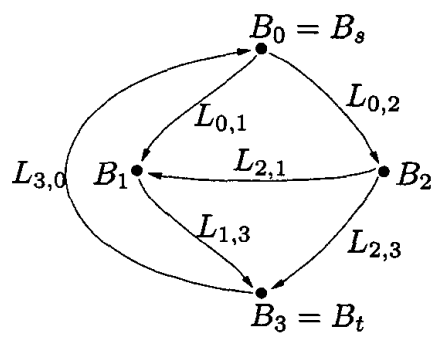

Figure 1: Example of a graph that cannot be reduced with only series and parallel reductions

The corresponding twist space is the support of the extensor formally obtained dualizing the expression of $L_{R_{\text {par }}}^{*}$ and using the property (7), that is,

$$
\mathbf{T}_{R_{p a r}}=\overline{L_{R_{p a r}}}=\overline{L_{0,1}^{1} \wedge L_{0,1}^{2} \wedge \cdots \wedge L_{0,1}^{k}} .
$$

Since the shuffle formula (4) expresses the twist space in terms of sum of extensors, in general, it gives rise to a tensor.

Similar considerations can be repeated for the wrench space of $R_{\text {ser }}$.

\subsection{Delta-Wye Transformations}

In some cases it is impossible to reduce a graph using only series and parallel reductions. This is the case of the fully connected graph represented in Fig. 1.

In general we also need delta-wye transformations [16] which consist in removing one nonterminal node of the graph and relating the nodes sharing an arc with it by means of a set of equivalent constraints.

Let us suppose that our graph has 4 nodes in wye configuration as represented in Fig. 2.a, we want to eliminate the nonterminal node $B_{4}$ and connect $B_{1} B_{2}$ and $B_{3}$ in delta configuration represented in Fig. 2.b in such a way that the constraints among them remain unchanged. So, the following conditions must be imposed

$$
\begin{aligned}
& L_{1,3}=L_{1,4} \vee L_{4,3} \\
& L_{2,3}=L_{2,4} \vee L_{4,3} \\
& L_{1,2}=L_{1,4} \vee\left(-L_{2,4}\right)=L_{1,4} \vee L_{4,2}
\end{aligned}
$$

If we have three nodes in a delta configuration as represented in Fig. 2.b and we want to transform it in a wye configuration, the expressions for the extensors $L_{1,4} L_{2,4}$ and $L_{4,3}$ cannot be obtained in a unique way by solving the above system of equations since its matrix has rank 2 . So, we must arbitrarily choose one of the extensors of the wye configuration, say 


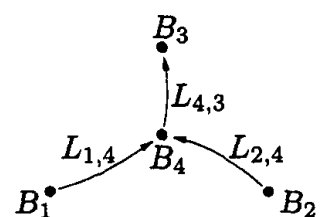

(a)

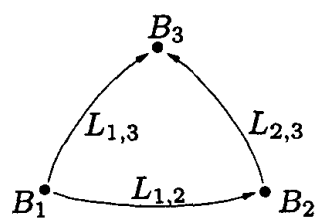

(b)
Figure 2: Wye and delta configurations

$L_{4,3}=-L_{3,4}$. The expressions of the other two extensors are

$$
\begin{aligned}
& L_{1,4}=L_{1,2} \vee L_{2,3} \vee\left(-L_{4,3}\right)=L_{1,2} \vee L_{2,3} \vee L_{3,4} \\
& L_{2,4}=L_{2,3} \vee\left(-L_{4,3}\right)=L_{2,3} \vee L_{3,4} .
\end{aligned}
$$

\subsection{A Case Study}

Consider the parallel manipulator represented in Fig. 3, where the spherical joints of the upper platform and the revolute joints of the lower one are both located at the vertices of an equilateral triangle [1.7].

The twist space $\mathbf{T}_{\boldsymbol{i}}$ of the leg $i$ of the manipulator is the support of the extensor $L^{i}=L_{r}^{i} \vee L_{p}^{i} \vee L_{s, 1}^{i} \vee$ $L_{s, 2}^{i} \vee L_{s, 3}^{i}$, where $L_{r}^{i}, L_{p}^{i}$, and $L_{s}^{i}$ are, respectively, the extensors of $r_{i}, p_{i}$ and $s_{i}$, the rotational, prismatic, and spherical joints of the leg $i$. The spherical joint $s_{i}$ is modelled as three revolute joints $s_{i, 1}, s_{i, 2}$ and $s_{i, 3}$ having intersecting axes whose extensors are, respectively, $L_{s, 1}^{i}, L_{s, 2}^{i}$ and $L_{s, 3}^{i}$.

The wrench space $\mathbf{W}_{i}$ of the leg $i$ is the support of the extensor $\left(L^{i}\right)^{*}$, dual of $L^{i}$.

The twist space $\mathbf{T}$ of the parallel manipulator is the intersection of the twist spaces of the three legs. Therefore, it can be can be expressed as the support of the meet of the corresponding extensors, that is, as the support of $L=L^{1} \wedge L^{2} \wedge L^{3}$. Using the associative property of the meet operator, we can compute first $L^{12}=L^{1} \wedge L^{2}$ and then $L^{12} \wedge L^{3}$. The shuffe formula (4) gives

$$
\begin{aligned}
& L^{12}=L^{1} \wedge L^{2}= \\
& +\left[L_{r}^{1} L_{r}^{2} L_{p}^{2} L_{s, 1}^{2} L_{s, 2}^{2} L_{s, 3}^{2}\right] L_{p}^{1} \vee L_{s, 1}^{1} \vee L_{s, 2}^{1} \vee L_{s, 3}^{1} \\
& -\left[L_{p}^{1} L_{r}^{2} L_{p}^{2} L_{s, 1}^{2} L_{s, 2}^{2} L_{s, 3}^{2}\right] L_{r}^{1} \vee L_{s, 1}^{1} \vee L_{s, 2}^{1} \vee L_{s, 3}^{1} \\
& +\left[L_{s, 1}^{1} L_{r}^{2} L_{p}^{2} L_{s, 1}^{2} L_{s, 2}^{2} L_{s, 3}^{2}\right] L_{r}^{1} \vee L_{p}^{1} \vee L_{s, 2}^{1} \vee L_{s, 3}^{1} \\
& -\left[L_{s, 2}^{1} L_{r}^{2} L_{p}^{2} L_{s, 1}^{2} L_{s, 2}^{2} L_{s, 3}^{2}\right] L_{r}^{1} \vee L_{p}^{1} \vee L_{s, 1}^{1} \vee L_{s, 3}^{1} \\
& +\left[L_{s, 3}^{1} L_{r}^{2} L_{p}^{2} L_{s, 1}^{2} L_{s, 2}^{2} L_{s, 3}^{2}\right] L_{r}^{1} \vee L_{p}^{1} \vee L_{s, 1}^{1} \vee L_{s, 2}^{1} \\
& =\sum_{i=1}^{5} \rho_{i} L_{i}^{12},
\end{aligned}
$$

where $L_{i}^{12}$ is the $i$-th 4 -extensor and $\rho_{i}$ is the value of the corresponding determinant.

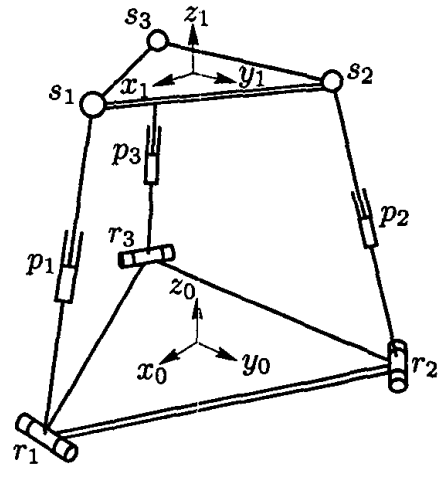

Figure 3: A parallel manipulator

The extensor $L^{12}$ describes the twist space of the subchain of the parallel manipulator composed by the legs 1 and 2. The twist space of this subchain is expressed as a function of the joint extensors of the leg 1. It is the sum of five 4-dimensional subspaces. The contribution of each subspace to the twist space of the end effector is weighted by the value of the corresponding determinant which depends on both the configurations of legs 1 and 2 . Since both $L^{1}$ and $L^{2}$ are 5-extensors, we have that $L^{2} \wedge L^{1}=-L^{1} \wedge L^{2}$. Therefore, the expression of the same twist space as a function of the extensors of the leg 2 can be obtained simply reversing the order of the operands and changing the sign of the result.

$L^{12}$ is expressed as a sum of 4-extensors, so that $L^{12} \wedge L^{3}$ will be the sum of 3 -extensors. Since the meet operation is distributive over addition we can write $L=L^{12} \wedge L^{3}=\sum_{i=1}^{5} \rho_{i} L_{i}^{12} \wedge L^{3}$ where, for example,

$$
\begin{aligned}
& L_{1}^{12} \wedge L^{3}= \\
& +\left[L_{p}^{1} L_{r}^{3} L_{p}^{3} L_{s, 1}^{3} L_{s, 2}^{3} L_{s, 3}^{3}\right] L_{s, 1}^{1} \vee L_{s, 2}^{1} \vee L_{s, 3}^{1} \\
& -\left[L_{s, 1}^{1} L_{r}^{3} L_{p}^{3} L_{s, 1}^{3} L_{s, 2}^{3} L_{s, 3}^{3}\right] L_{p}^{1} \vee L_{s, 2}^{1} \vee L_{s, 3}^{1} \\
& +\left[L_{s, 2}^{1} L_{r}^{3} L_{p}^{3} L_{s, 1}^{3} L_{s, 2}^{3} L_{s, 3}^{3}\right] L_{p}^{1} \vee L_{s, 1}^{1} \vee L_{s, 3}^{1} \\
& -\left[L_{s, 3}^{1} L_{r}^{3} L_{p}^{3} L_{s, 1}^{3} L_{s, 2}^{3} L_{s, 3}^{3}\right] L_{p}^{1} \vee L_{s, 1}^{1} \vee L_{s, 2}^{1} .
\end{aligned}
$$

The meet between $L_{i}^{12}, i=2, \ldots, 5$, and $L^{3}$ can be computed in a similar way. This means that the support of $L$ is a 3 -tensor and, hence, the moving platform has three degrees of freedom. Using the same geometric parameters as in [17] it is possible to deduce that the upper platform has two degrees of freedom in orientation and one in Cartesian position.

By duality the wrench space can be easily obtained. In some cases, given the joint extensors, it is convenient to compute first $L^{*}=\left(L_{1} \wedge L_{2} \wedge L_{3}\right)^{*}=$ $L_{1}^{*} \vee L_{2}^{*} \vee L_{3}^{*}$ whose support is $\mathbf{W}$ and then, by duality, T. 
Other examples of application of the GrassmannCayley algebra to the kinestatic analysis of robot manipulators can be found in [8].

\section{Conclusions}

In this paper a unifying framework for the study of the statics and the instantaneous kinematics of robot manipulators based on the Grassmann-Cayley algebra has been presented. It has been shown that the Grassmann-Cayley algebra permits the mathematical representation of all the basic concepts of screw theory. Twist and wrench spaces of partially constrained rigid bodies are represented by means of extensors and the reciprocity condition between them is reflected by the inherent duality of this algebra. Moreover, its join and meet operator are used to implement, respectively, the operations of sum and intersection of linear subspaces in the kinestatic analysis on manipulators. Of special relevance is the existence of an explicit formula for the meet operator that permits to obtain analytical expressions of the twist and wrench spaces of robot manipulators with arbitrary topology.

\section{References}

[1] R. S. Ball, A Treatise on the Theory of Screws. Cambridge University Press, 1900. Reprint 1998.

[2] K. H. Hunt, Kinematic Geometry of Mechanisms. Oxford Science Publications, 1990.

[3] J. Duffy, "The fallacy of modern hybrid control theory that is based on "orthogonal complements" of twist and wrench spaces," Journal of Robotic Systems, vol. 7, no. 2, pp. 139-144, 1990.

[4] E. Staffetti and F. Thomas, "Kinestatic analysis of serial and parallel robot manipulators using Grassmann-Cayley algebra," in Advances in Robot Kinematics (J. Lenarčič and M. M. Stanišić, eds.), Kluwer Academic Publishers, 2000.

[5] D. Kim, W. Chung, and Y. Youm, "Analytic Jacobian of in-parallel manipulators," in Proceedings of the 2000 IEEE International Conference on Robotics and Automation, 2000.

[6] S. Dutré, H. Bruyninckx, and J. D. Schutter, "The analytic Jacobian and its derivative for a parallel manipulator," in Proceedings of the 1997 IEEE International Conference on Robotics and Automation, 1997.

[7] E. Staffetti and F. Thomas, "Analysis of rigid body interactions for compliant motion tasks using the Grassmann-Cayley algebra," in Proceedings of the
IEEE/RSJ International Conference on Intelligent Robotic Systems, 2000.

[8] E. Staffetti, Local Characterization of the Configuration Space. PhD thesis, Universitat Politècnica de Catalunya, Barcelona, Spain, 2001. Available at http://www-iri.upces/people/staffeti/thesis.html.

[9] N. White, "Grassmann-Cayley algebra and robotics," Journal of Intelligent Robotics Systems, vol. 11, pp. 91-107, 1994.

[10] H. Crapo and W. Whiteley, "Static of frameworks and motions of panel structures, a projective geometric introduction," Structural Topology, vol. 6, pp. 43-82, 1982.

[11] M. Barnabei, A. Brini, and G.-C. Rota, "On the exterior calculus of invariant theory," Journal of Algebra, vol. 96, pp. 120-160, 1985.

[12] N. White, "A tutorial on Grassmann-Cayley algebra," in Invariant Methods in Discrete and Computational Geometry (N. White, ed.), pp. 93-106, Kluwer Academic Publisher, 1995.

[13] W. V. D. Hodge and D. Pedoe, Methods of Algebraic Geometry. Cambridge University Press, 1994.

[14] G. Golub and C. Van Loan, Matrix Computations. The Johns Hopkins University Press, 1989.

[15] H. Bruyninckx, S. Demey, S. Dutré, and J. De Schutter, "Kinematic models for model-based compliant motion in the presence of uncertainty," The International Journal of Robotics Research, vol. 14, no. 5, pp. 465-482, 1995.

[16] T. A. Feo and J. S. Provan, "Delta-wye transformation and the efficient reduction of two-terminal planar graphs," Operations Research, vol. 41, no. 3, pp. 572$582,1993$.

[17] K.-M. Lee and D.-K. Shah, "Kinematic analysis of a three-degrees-of-freedom in-parallel actuated manipulator," IEEE Journal of Robotics and Automation, vol. 4, no. 3, pp. 354-360, 1988. 